\title{
Update on the role of trabectedin in the treatment of intractable soft tissue sarcomas
}

\author{
This article was published in the following Dove Press journal: \\ OncoTargets and Therapy \\ 23 February 2017 \\ Number of times this article has been viewed
}

Federica Recine
Alberto Bongiovanni
Nada Riva
Valentina Fausti
Alessandro De Vita
Laura Mercatali
Chiara Liverani
Giacomo Miserocchi
Dino Amadori
Toni Ibrahim
Osteoncology and Rare Tumors
Center, Istituto Scientifico Romagnolo
per lo Studio e la Cura dei Tumori
(IRST) IRCCS, Meldola, Italy

Correspondence: Alberto Bongiovanni Osteoncology and Rare Tumors Center, Istituto Scientifico Romagnolo per lo Studio e la Cura dei Tumori (IRST) IRCCS, Via Piero Maroncelli 40, 470I4 Meldola (FC), Italy

Tel +3905 43739100

Fax +3905 43739123

Email alberto.bongiovanni@irst.emr.it

\begin{abstract}
Soft tissue sarcomas (STS) represent a variety of tumors of mesenchymal origin, accounting for about $1 \%$ of all adult cancers. This group of tumors comprises over 60 different histotypes with different biology showing different sensitivity to therapeutic agents. For decades, the standard first-line systemic treatment of metastatic STS has comprised anthracycline basedchemotherapy. Second-line therapy options include agents such as ifosfamide, gemcitabine, and pazopanib, but the optimal sequential therapy for the management of metastatic disease has yet to be defined. Trabectedin is one of the new molecules approved for patients in progression after first-line chemotherapy with anthracyclines or for those unfit for these agents. The compound is characterized by multiple potential mechanisms of action combining cytotoxic, targeted, and immunological effects. This article takes an in-depth look at the role of trabectedin in the management of metastatic STS, including L-sarcoma and non-L-sarcoma.
\end{abstract}

Keywords: soft tissue sarcoma, histotypes, chemotherapy, L-sarcomas, non-L-sarcomas

\section{Introduction}

Soft tissue sarcomas (STS) are a group of rare, highly heterogeneous tumors of mesenchymal origin whose incidence is about $1 \%$ of all solid tumors worldwide. ${ }^{1}$ The latest World Health Organization classification recognizes the existence of over 60 different histotypes of STS. Among these, the most common variants are liposarcoma (LPS) and leiomyosarcoma (LMS), both of which show specific clinical and biologic features. ${ }^{2,3}$

Although STS can develop in any mesenchymal site of the body, the extremities and retroperitoneum represent the most frequent localizations, occurring in $60 \%$ and $20 \%$ of cases, respectively. ${ }^{4}$ Optimal management of STS is challenging and requires a multimodality therapeutic approach involving a multidisciplinary team from STS reference centers. ${ }^{5}$

Wide surgery is the cornerstone of treatment for localized disease, whereas the role of adjuvant and neoadjuvant therapies remains controversial and is only proposed in selected cases. ${ }^{6}$ However, a considerable number of patients $(30 \%-50 \%)$ will develop metastatic disease, with an overall survival (OS) of about 12-15 months. ${ }^{7}$

In the metastatic setting, palliative systemic anthracycline-based chemotherapy with or without ifosfamide represents the standard first-line treatment. ${ }^{8}$ Second- or further-line chemotherapy including gemcitabine, dacarbazine or ifosfamide can also be used on the basis of the sarcoma histotype, but the optimal sequencing strategy of therapy has yet to be identified. ${ }^{9,10}$ Within the group of targeted therapies, pazopanib is an option for the management of previously treated nonadipocytic sarcomas. ${ }^{11}$ Moreover, another drug, eribulin, was recently approved for use in pretreated patients with 
adipocytic sarcomas. ${ }^{12}$ However, there are still no molecular biomarkers to optimize the available treatments and to overcome the extremely biological and clinical heterogeneity of STS. In this scenario, trabectedin has an important role, showing multiple mechanisms of action including cytotoxic and antiangiogenic effects. Furthermore, trabectedin represents an innovative treatment option approved in Europe for patients with metastatic STS who are unfit for anthracyclines or who progress after therapy with these drugs.

The latest European Society for Medical Oncology (ESMO) guidelines proposed trabectedin as a second-line option in STS patients, ${ }^{5}$ and the drug has proven to be effective, especially in LMS and LPS histotypes, now commonly referred to collectively as L-sarcomas. ${ }^{5,13,14}$

Results from some retrospective and randomized trials also report the antitumor activity of trabectedin in non-L-type sarcomas. Furthermore, some small studies have shown the potential usefulness of the drug for the treatment of specific non-L-type sarcoma histotypes such as synovial sarcoma and solitary fibrous tumors (SFTs). ${ }^{15-18}$ Trabectedin has also been approved for the treatment of ovarian cancer.

This article represents an overview of the role of trabectedin in the management of metastatic STS, including non-Ltype sarcoma, and provides evidence of the activity of this drug in rare and intractable histological subtypes.

\section{L-sarcomas}

LPS and LMS are among the most common mesenchymal sarcomas, accounting for $15 \%$ and $11 \%$ of all adult STS, respectively. Despite the heterogeneity of their histology, biology, and clinical behavior, LPS and LMS are collectively referred to in the literature as L-sarcomas. ${ }^{4}$ LPS are a heterogeneous group of adipocytic neoplasms originating from adipocytes that can occur anywhere in the body but are found mainly in the retroperitoneum (45\%) and extremities (24\%). Several factors such as histologic subtype and tumor location can predict patient outcome. Indeed, it has been recognized that risk of recurrence is higher for patients with dedifferentiated histology or a retroperitoneal location. ${ }^{19}$

Histologically, LPS is classified into several subtypes: well-differentiated liposarcoma/atypical lipomatous tumor (WDLPS/ALT), dedifferentiated liposarcoma (DDLPS), myxoid/round cell liposarcoma (MLPS), and pleomorphic liposarcoma (PLS). Each of these subtypes is extremely different in terms of biology, clinical course, and sensitivity to drugs. ${ }^{20}$

WDLPS/ALT, accounting for $40 \%-45 \%$ of all LPS, is characterized by an indolent biology with almost no metastatic potential but a high tendency to recur locally. Morphologically, it is composed of mature adipocytes and can be classified into 3 further subtypes: adipocytic, sclerosing, and inflammatory. In $25 \%-40 \%$ of WDLPS/ALTs, tumor dedifferentiation may occur upon relapse, indicating a more aggressive phenotype with a higher tendency to metastasize. $^{21}$

DDLPS, occurring in $25 \%$ of LPS, shows more aggressive behavior and has a high metastatic rate of about $20 \%-30 \%$. This LPS subtype is correlated with an overall mortality of $50 \%-75 \%$. Histologically, DDLPS is characterized by the presence of a non-lipogenic component and can appear ex novo or after tumor progression from WDLPS. ${ }^{22}$

The majority of WDLPS/ALTs and DDLPS show MDM2 amplification or overexpression, which represents the key marker for the diagnosis of these tumors and is involved in cell transformation and in the tumorigenesis process. ${ }^{23}$

MLPS is the second-most common LPS subtype, representing about $30 \%$ of all LPS. It is histologically characterized by the presence of spindle or ovoid cells and signet ring lipoblasts within a myxoid stroma. The presence of round cells is correlated with dedifferentiation and a poorer prognosis. This sarcoma histotype shows a peculiar propensity to develop metastases in soft tissues, serosal membranes, and bones rather than the lungs. MLPS is also an indolent disease but has a more unpredictable clinical behavior than that of WDLPS. The most common sites for primary MLPS are the extremities and, rarely, the retroperitoneum. However, it can potentially occur in any anatomical location. MLPS normally responds better to chemotherapy than other STS. ${ }^{24}$

Oncogenic mechanisms have been associated with the pathogenesis of various sarcoma subtypes. MLPS is characterized by the chromosome translocation $\mathrm{t}(12 ; 16)$ (q13;p11) in over than $95 \%$ of cases, resulting in a specific fusion gene called FUS-CHOP gene. Chromosome translocation $\mathrm{t}(12 ; 22)(\mathrm{q} 13 ; \mathrm{q} 12)$ occurs less frequently, resulting in the formation of the EWS-CHOP oncogene. These alterations are considered the primary oncogenic events in sarcoma tumorigenesis. . $^{25,26}$

PLS is a variant of LPS, occurring in $5 \%-15 \%$ of cases and affecting mainly the lower extremities. It is associated with a poor prognosis and, given its rarity, there is little information available about treatment in the metastatic setting. PLS is often resistant to chemotherapy. ${ }^{27}$

Conversely, LMS represent a group of heterogeneous tumors deriving from smooth muscle cells, with an incidence of $11 \% .{ }^{4}$ Although they can occur anywhere in the body, the uterus is the most common site of disease, representing about 
$50 \%$ of all LMS. Based on the anatomic localization, LMS is divided into 2 groups: uterine and extrauterine LMS. LMS of the uterus is characterized by aggressive clinical behavior with a predilection for early hematogenous spread and the development of lung metastases. Extrauterine LMS can occur in different anatomic areas, including bone, retroperitoneum, blood vessels, skin, and less frequently liver and thyroid. ${ }^{28}$

All of these subgroups exhibit some common immunohistochemical markers, including smooth muscle actin, desmin, caldesmon, and, less frequently, S-100. ${ }^{2}$ They are characterized by highly variable clinical behavior reflecting distinct disease biologies, with a high tendency to develop distant metastases to the lung and less commonly to the liver, bone, and soft tissues. ${ }^{29}$

\section{Current management strategies}

Radical surgical resection is the cornerstone of treatment with curative intent for localized disease. Although complementary treatments to surgery including radiotherapy and chemotherapy are still controversial, they can be taken into consideration for specific cases selected on the basis of factors such as tumor site and histology. ${ }^{6}$

Despite adequate surgical resection, the majority of patients with STS remain at high risk for local and distant metastases. ${ }^{5,19}$ Initial treatment for patients with unresectable metastatic STS includes standard cytotoxic chemotherapy comprising anthracyclines with or without ifosfamide. Higher response rates $(26 \%)$ have been observed for combination treatments than for single-agent therapy $(14 \%) .{ }^{8}$ Although several treatment options are available after failure of firstline chemotherapy, the optimal standard sequential therapy has yet to be defined. Second-line therapies include cytotoxic drugs, such as ifosfamide, dacarbazine, gemcitabine, and taxanes, but data from randomized trials on single agents are lacking. ${ }^{5,30}$ Interesting phase II results have been obtained on combination regimens such as gemcitabine plus dacarbazine, or gemcitabine plus docetaxel., ${ }^{9,31}$

Several agents have been identified for the treatment of L-sarcoma histotypes on the basis of the new concept of histology-tailored therapy. Pazopanib, a multitargeted tyrosine kinase inhibitor, has been shown to improve progression-free survival (PFS) in patients with STS compared to placebo, with the exception of adipocytic sarcomas in which the drug failed to produce a clear benefit. ${ }^{11}$

New drugs such as trabectedin and eribulin have recently proven to be more effective than dacarbazine in randomized trials on advanced sarcoma. In particular, eribulin is now approved for the treatment of adipocytic sarcomas on the basis of the improvement in OS obtained in this subset of sarcomas. $^{12}$

\section{Trabectedin}

\section{Pharmacologic profile}

Trabectedin (Yondelis ${ }^{\circledR}$, Ecteinascidin, ET-743) is a marinederived anticancer agent consisting of a tetrahydroisoquinoline molecule that was originally isolated from the Caribbean sea sponge Ecteinascidia turbinata. It is currently produced synthetically by PharmaMar Pharmaceutical Industry. Trabectedin exerts multitargeted and pleiotropic antitumor activities including cytotoxic and antiproliferative effects, inhibition of gene transcription, and indirect immunologic and antiangiogenic actions. ${ }^{32}$ However, the mechanisms of action of the drug are still not completely understood.

Molecular evidence suggests that the cytotoxic effects of trabectedin are a result of its binding to DNA. In fact, trabectedin binds to the DNA minor groove, causing DNA double-helix distortion with breaks in the DNA itself. The interaction between trabectedin and the DNA minor groove determines structural changes in the molecule, which is a cascade of events that affects several transcription factors, DNA-binding proteins, and DNA repair pathways, resulting in $\mathrm{G}_{2}-\mathrm{M}$ cell cycle arrest and ultimately apoptosis. ${ }^{33,34}$

It has been observed that the cytotoxic effect of trabectedin is influenced by DNA repair mechanisms such as nucleotide excision repair (NER) and homologous recombination repair (HRR), both of which recognize the DNA damage and recruit various factors to repair the damaged site. The cell repair machinery, including both NER and HRR systems, is crucial for the interaction between trabectedin and DNA and appears to be the most important determinant of sensitivity to the drug. ${ }^{35}$ Notably, a direct interaction has been reported between trabectedin and RNA polymerase II (Pol II) factor, causing the arrest of the transcription process, the degradation of Pol II by the proteasome pathway, and the premature termination of the RNA transcript. ${ }^{34}$ This kind of antiproliferative mechanism appears to be especially effective in MLPS, which represents the most responsive sarcoma subtype to trabectedin. Furthermore, trabectedin has shown a pro-differentiation effect in tumor cells from MLPS. Tumor response to trabectedin in MLPS in vivo is characterized by tumor cell depletion and induction of mature adipocytes. ${ }^{36}$

In addition to these cytotoxic activities, trabectedin modulates the tumor microenvironment and this seems to be the most important part of its therapeutic effect. The drug exerts a selected cytotoxic effect against monocytes and tumor-associated macrophages (TAMs) present in several 
tumor tissues, which are the key promoters of tumor-related inflammation. TAMs exhibit pro-tumoral effects, including the production of several growth factors that are essential for neoangiogenesis proliferation and proteolytic enzymes. These elements degrade the extracellular matrix, determining neoplastic cell invasion and facilitating immune control escape.

It has been demonstrated that trabectedin induces a significant downregulation of cytokines, chemokines, and inflammatory and angiogenic mediators, eg, interleukin-6, chemokine ligand-2, and vascular endothelial growth factor, which, in turn, modify the tumor microenvironment, contributing to the antitumor and antiangiogenic effects of trabectedin. ${ }^{37}$

\section{Clinical evidence of trabectedin activity}

The clinical activity of trabectedin has been demonstrated in several clinical studies, leading to its approval in 2007 by the European Medicines Agency for the treatment of patients with advanced STS following failure of first-line chemotherapy or as first-line treatment for patients unfit for anthracycline-based treatment. In 2009, the drug was also approved for patients with relapsed platinum-sensitive ovarian cancer in combination with pegylated liposomal doxorubicin. In 2015, the U.S. Food and Drug Administration approved the use of trabectedin for the treatment of unresectable or metastatic LPS and LMS. ${ }^{13,15,16,38}$ The drug is also currently under evaluation in prostate and breast cancer.

Phase I clinical trials carried out to assess the optimal dose and various schedules of trabectedin identified 2 different schedules ( 3 hours and 24 hours in continuous infusion) in which the main dose-limiting toxicities were neutropenia, fatigue, and thrombocytopenia, while the maximum tolerated dose was $1.8 \mathrm{mg} / \mathrm{m}^{2}$ in either schedule. The trials also selected 3 regimens of trabectedin comprising $1.3 \mathrm{mg} / \mathrm{m}^{2}$ in a 3-hour infusion every 3 weeks, $1.5 \mathrm{mg} / \mathrm{m}^{2}$ in a 24 -hour infusion every 3 weeks, and $0.58 \mathrm{mg} / \mathrm{m}^{2}$ in a 3 -hour infusion each week for 3 out of 4 weeks. These doses were used in the subsequent phase II trials, leading to the current optimal trabectedin administration of $1.5 \mathrm{mg} / \mathrm{m}^{2}$ as a 24 -hour continuous infusion every 3 weeks. ${ }^{39-42}$

\section{Trabectedin in L-sarcoma}

Several clinical trials assessed the role of trabectedin in patients with metastatic LMS and LPS. Results from phase II trials showed low overall response rates (ORRs) but good disease control and better survival in previously treated patients and in those refractory to treatment with anthracycline-based chemotherapy. ${ }^{43,44}$ In particular, a phase II trial reported response rates of $8 \%$ and a 6-month and 1-year survival of
$74 \%$ and $53 \%$, respectively, in 36 previously treated sarcoma patients. Interestingly, the best responses lasted for more than 20 months. $^{43}$

Based on these results, the European Organization for the Research and Treatment of Cancer (EORTC) conducted a phase II trial in a larger case series, reporting that the use of trabectedin in 104 heavily pretreated STS patients was correlated with prolonged OS, suggesting a benefit of the drug in terms of tumor control and long-lasting disease stabilization. The most common histotype in the trial was LMS, accounting for $41 \%$ of all cases, while $10 \%$ of patients had LPS. ${ }^{45}$

Demetri et al performed a multicenter, randomized phase II trial (STS-201 study) on 270 pretreated L-sarcoma patients to compare the safety and efficacy of trabectedin in 2 different schedules, $1.5 \mathrm{mg} / \mathrm{m}^{2}$ over 24 hours every 3 weeks and $0.58 \mathrm{mg} / \mathrm{m}^{2}$ over 3 hours each week for 3 out of 4 weeks. Results showed a significant $(P=0.0302)$ reduction in disease progression (27\%) with trabectedin $1.5 \mathrm{mg} / \mathrm{m}^{2}$ administered over 24 hours every 3 weeks, but no significant differences in OS or toxicity. Notably, a 1-year OS rate of $60 \%$ was observed for patients receiving the 24-hour infusion schedule. ${ }^{14}$

The subsequent randomized, open-label, phase III trial, evaluated trabectedin vs dacarbazine in 518 previously treated patients with metastatic L-sarcoma. The study demonstrated a significant improvement in PFS in the trabectedin arm (4.2 vs 1.5 months), with the highest benefit observed in patients with MLPS and a $45 \%$ reduction in the risk of disease progression or death. No differences in OS (13.7 vs 13.1 months) were seen between the 2 arms. ${ }^{13}$ Of note, the reported superiority of trabectedin over dacarbazine occurred in LMS (uterine and non-uterine) and in all LPS subtypes. However, there is still some debate as to whether the results from the trial can be considered valid for all LPS subtypes, especially DDLPS. ${ }^{3}$

Following the approval of trabectedin, a worldwide expanded access program (EAP) study and compassionate use trial were performed on larger and unselected populations of metastatic patients with STS to evaluate the role of the drug in "more realistic" clinical situations. The EAP study by Samuels et al, which included patients with relapsed STS (61\% were L-sarcomas), aimed to assess clinical outcomes and the safety of trabectedin in a "real world" population. ${ }^{46}$ Blay et al performed a multicenter compassionate use trial on 181 French patients with a variety of STS (57\% were L-sarcomas), treated with trabectedin as first- and further-line chemotherapy. ${ }^{47}$ Results from both the EAP and the compassionate use studies revealed higher ORRs and longer PFS and OS in L-sarcoma patients, especially those with MLPS, 
than other histotypes. Interestingly, both study populations included around $20 \%$ of elderly patients $(19 \% \geq 65$ years and $20 \%>60$ years in Samuels and Blay's case series, respectively), but no significant differences were observed in outcome compared to younger patients.

Trabectedin has been found to be particularly effective in translocation-related sarcomas (TRS) such as MLPS, exerting an important antitumor activity probably through the inactivation of the FUS-CHOP oncogene, which is thought to alter gene-encoding protein expression and induce lipogenic cell differentiation. ${ }^{29,36,38}$ A retrospective multicenter analysis of 51 previously treated patients with metastatic MLPS given trabectedin in a compassionate-use program reported 2 complete response (CR) and 24 partial response (PR) with long-lasting tumor control after a median follow-up of 14 months. Results showed an ORR of $51 \%$, an overall tumor control rate of $90 \%$, and a median PFS of $88 \% .^{38}$ A phase II trial evaluated the use of trabectedin in 23 patients with untreated locally advanced MLP; 3 patients achieved pathological CR, 7 obtained PR according to RECIST, and 12 showed a moderate-to-good histological response. There was an overall disease control rate (DCR) of $96 \%$, with no cases of disease progression. These findings suggest that trabectedin could be a promising therapeutic option in the neoadjuvant setting in selected patients with this sarcoma histotype. ${ }^{48}$

An international phase III study of localized high-risk STS of the extremities and trunk wall (TAILORED trial) is currently ongoing to compare standard neoadjuvant chemotherapy comprising anthracycline and ifosfamide with histology-tailored therapy consisting specifically of trabectedin for patients with MLPS (NCT01710176). Preliminary results presented at the ESMO 2016 Annual Meeting showed that 3 full-dose cycles of neoadjuvant treatment with an anthracycline plus ifosfamide correlated with a $20 \%$ benefit in relapse-free survival and OS with respect to a different chemotherapy regimen in high-risk STS patients. Although the study did not highlight any benefit from histology-driven chemotherapy, a subgroup analysis suggested that patients with high-grade MLPS treated with trabectedin had similar PFS and OS than those treated with epirubicin plus ifosfamide. ${ }^{49}$

\section{Trabectedin in non-L-type sarcoma}

Trabectedin has been shown to be active in a variety of histological subtypes, including non-L-type sarcoma, especially when characterized by chromosomal translocation $(20 \%$ of all sarcomas). These translocations generate chimeric oncoproteins, which act as abnormal transcription factors. ${ }^{50}$ The most common TRS are listed in Table $1 .^{51-56} \mathrm{~A}$ number of randomized clinical trials and retrospective analyses have been conducted in this area to explore the role of trabectedin in these poorly understood sarcoma histotypes. Some focus on specific histologies, such as synovial sarcoma and SFT. ${ }^{77,58}$

The phase II trial conducted by EORTC in 2012 reported a considerable number of non-L-sarcoma histologies, including

Table I The most frequent chromosomal translocations and genetic abnormalities in sarcomas

\begin{tabular}{|c|c|c|c|}
\hline Histology & Incidence (\%) & Chromosomal/genetic abnormalities & Genes involved \\
\hline \multirow[t]{2}{*}{ Myxoid liposarcoma ${ }^{24,25}$} & \multirow[t]{2}{*}{10} & $\mathrm{t}(|2 ;| 6)(q|3 ; p| \mathrm{l})$ & TLS-CHOP \\
\hline & & $\mathrm{t}(\mid 2 ; 22)(\mathrm{q}|3 ; \mathrm{q}| 2)$ & EWS-CHOP \\
\hline Desmoplastic small round cell tumor ${ }^{50}$ & I & $\mathrm{t}(I \mid ; 22)(p|3 ; q| 2)$ & EWS-WTI \\
\hline Alveolar soft part sarcoma ${ }^{51}$ & I & $t(X ; \mid 7)(p|| .2 ; q 25)$ & TFE3-ASPL \\
\hline Ewing's sarcoma/peripheral primitive & \multirow[t]{6}{*}{ I } & $\mathrm{t}(\mathrm{I} \mid 22)(q 24 ; q \mid 2)$ & EWS-FLII \\
\hline \multirow[t]{5}{*}{ neuroectodermal tumor ${ }^{52}$} & & $\mathrm{t}(21 ; 22)(q 22 ; q \mid 2)$ & EWS-ERG \\
\hline & & $t(7 ; 22)(p 22 ; q 12)$ & EWS-ETVI \\
\hline & & $\mathrm{t}(\mid 7 ; 22)(q|2 ; q| 2)$ & EWS-FEV \\
\hline & & $\mathrm{t}(2 ; 22)(\mathrm{q} 33 ; \mathrm{q} \mid 2)$ & EWS-EIAF \\
\hline & & $\mathrm{t}(\mid 6 ; 21)(p \mid I ; q 22)$ & FUS-ERG \\
\hline Dermatofibrosarcoma protuberans ${ }^{53}$ & I & $\mathrm{t}(\mid 7 ; 22)(q 22 ; q \mid 3)$ & COLIAI-PDGFB \\
\hline \multirow[t]{3}{*}{ Extraskeletal myxoid chondrosarcoma ${ }^{54}$} & \multirow[t]{3}{*}{ I } & $\mathrm{t}(9 ; 22)(\mathrm{q} 22 ; \mathrm{q} / 2)$ & EWS-CHN \\
\hline & & $\mathrm{t}(9 ; 15)(\mathrm{q} 22 ; \mathrm{q} 21)$ & TFCI 2-CHN \\
\hline & & $\mathrm{t}(9 ; 17)(\mathrm{q} 22 ; \mathrm{q} \mid \mathrm{I})$ & TAF2N-CHN \\
\hline \multirow[t]{2}{*}{ Clear cell sarcoma ${ }^{55}$} & \multirow[t]{2}{*}{$<1$} & \multirow[t]{2}{*}{$\mathrm{t}(\mid 2 ; 22)(q|3 ; q| 2)$} & EWSRI/CREBI \\
\hline & & & EWSRI/ATFI \\
\hline \multirow[t]{3}{*}{ Inflammatory myofibroblastic tumor ${ }^{56}$} & \multirow[t]{3}{*}{$<1$} & \multirow[t]{3}{*}{$t(q 2 ; p 23)$} & $E M L 4-A L K$ \\
\hline & & & RANBP2-ALK \\
\hline & & & TFG-ROSI \\
\hline Solitary fibrous tumor ${ }^{57}$ & I & $\mathrm{t}(9 ; 22)(q 3|; p| 3)$ & NAB2-STAT6 \\
\hline Synovial sarcoma ${ }^{58}$ & $6-10$ & $t(X ; \mid 8)(p \mid I ; q I I)$ & SYT-SSX \\
\hline Pleomorphic sarcoma/undifferentiated sarcoma ${ }^{26}$ & $15-25$ & Genomic instability/complex karyotype & - \\
\hline
\end{tabular}


synovial sarcoma $(17.3 \%)$ in which trabectedin achieved response rates of up to $61 \% .{ }^{45}$ Blay et al performed a firstline, phase III randomized trial comparing trabectedin with doxorubicin-based chemotherapy in a population of 121 patients with STS, $88 \%$ of whom had TRS. The study did not show significant differences in PFS (hazard ratio [HR] $=0.86 ; 95 \%$ confidence interval [CI]: $0.4-1.8, P=0.6992$ ) or OS between the 2 groups (OS $=63.9 \%$ for trabectedin vs $58.3 \%$ for doxorubicin; $\mathrm{HR}=0.77 ; 95 \% \mathrm{CI}: 0.4-1.4, P=0.3672$ ). Response rates according to RECIST were higher in patients treated with doxorubicin ( $27 \%$ vs $5.9 \%$ ) but similar between treatment arms according to Choi criteria (45.9\% vs $37.3 \%$ ). ${ }^{59}$

A multicenter, randomized, controlled, open-label study was conducted in Japan on 73 pretreated TRS patients including 19\% synovial sarcoma, 8\% mesenchymal chondrosarcoma, and $8 \%$ extraskeletal Ewing's sarcoma/primitive neuroectodermal tumor $(8 \%)$, all refractory to standard chemotherapy and treated with trabectedin. Median PFS was 5.6 months (95\% CI: 4.1-7.5) in patients receiving trabectedin compared to 0.9 months (95\% CI: $0.7-1.0)$ in the best supportive care (BSC) group. The authors' concluded that trabectedin had a higher clinical benefit than that of BSC in these types of sarcomas. ${ }^{60}$

A French retrospective pooled analysis performed to assess the efficacy of trabectedin in 81 patients with different TRS subtypes reported a 10\% ORR, with a 59\% DCR. The most frequent STS histology in this trial was synovial sarcoma (around 56\%), patients showing a median PFS of 3 months, a 6-month PFS rate of 22\% (95\% CI: 9\%-34\%), a median OS of 13.9 months (95\% CI: 7.2-19.4), and a 2-year survival rate of $28 \%$ (95\% CI: $13 \%-43 \%$ ). Notably, OS results were similar to those reported in the STS-201 study on L-sarcoma. ${ }^{16}$

In addition, an Italian retrospective study analyzed 72 patients with metastatic pretreated STS given trabectedin to evaluate the activity of the drug in different STS histotypes. In addition to confirmation of its effectiveness in metastatic LPS and LMS, further evidence of its activity was also seen in other histologies, such as synovial sarcoma and high-grade undifferentiated pleomorphic sarcoma. The median PFS and OS were 2.97 months and 16.5 months, respectively, with a median follow-up time of 11 months. ${ }^{18}$

A German single-center retrospective study assessed the use of trabectedin in 101 elderly or heavily pretreated patients with different types of sarcoma, reporting significant activity of the drug in the subgroup with non-L-sarcomas including synovial sarcoma, pleomorphic sarcoma, and rhabdomyosarcoma. In particular, 22\% were classified as "other sarcomas", comprising alveolar sarcoma, chondrosarcoma, desmoplastic small round-cell tumor, epithelioid sarcoma, fibromyxoid sarcoma, MPNST, hemangiopericytoma, and not otherwise specified sarcoma. The clinical benefit rate (defined as CR or PR or stable disease for at least 6 weeks) in the non-Lsarcoma group was 35\% with a median PFS of 1.6 months. Long-lasting disease stabilization was observed in the rhabdomyosarcoma histology group. This trial also confirmed that administration of trabectedin is well tolerated in the elderly and heavily pretreated patients. ${ }^{61}$

A retrospective analysis carried out by Sanfilippo et al focused on the role of trabectedin in 61 patients with pretreated metastatic synovial sarcoma. There were 9 (15\%) PR according to RECIST criteria and 21 (35\%) stable disease (SD), with a DCR of $50 \%$. The 6 -month PFS was $23 \%$ and the median PFS was 3 months. ${ }^{17}$

There is evidence to suggest that trabectedin could also be a valid option for the SFT subtype. A case report by Chaigneau et al described the role of this drug in a young patient with lung and liver metastases from SFT of the pleura. After 4 cycles of trabectedin, the patient showed a PR in both the lung and liver. Disease progression occurred 8 months after the start of trabectedin. ${ }^{62}$

The French Sarcoma Group performed a retrospective analysis of trabectedin administered to 11 patients with pretreated metastatic SFT, reporting 1 PR (9.1\%) and $8(72.7 \%)$ SD. The DCR was $81.8 \%$. With the exception of 1 patient, SD and PR were further confirmed at 6 months. The median time to progression was 5.6 months (range: 1.9-25.3 months). These data suggest the potential usefulness of trabectedin in metastatic SFT. ${ }^{15}$

The role of trabectedin as first-line chemotherapy of treatment-naïve patients with non-L-sarcoma has also been investigated. A multicenter, randomized, prospective dose-selection phase IIb superiority clinical trial (TRUSTS trial) compared the efficacy of first-line trabectedin using 2 different schedules (3- and 24-hour infusion) with that of doxorubicin in 133 patients with metastatic STS. The study was initially planned as a combined phase IIb/III study. Preliminary data revealed a lack of superiority of either trabectedin schedule over doxorubicin in terms of ORR, PFS, and OS. Based on these results, the study was discontinued after the phase IIb part of the research. ${ }^{63}$

Another randomized phase II trial compared doxorubicin with trabectedin (experimental arm) vs doxorubicin alone (control arm) for the first-line treatment of advanced STS, including patients with non-L-sarcoma. The main endpoint of the trial was PFS. The study reported negative 
results (median PFS 5.7 months in the experimental arm vs 5.5 months in the control arm), demonstrating that a firstline trabectedin-doxorubicin combination was not superior to doxorubicin alone in this disease setting. ${ }^{64}$ In addition, the association of trabectedin with doxorubicin is limited due to cumulative toxicity. The results from the main clinical trials of trabectedin are reported in Table 2.

\section{Safety considerations}

Phase II and III trials have shown that trabectedin used as a single agent is not correlated with any grade of toxicity in about $91 \%$ of cases, with grade 3 and 4 adverse events occurring in only about $10 \%$ of cases. The most common grade 3 and 4 side effects are a reversible increase in transaminase levels and hematotoxicity, in particular neutropenia and anemia. Transient elevated transaminase values usually occur a few days after the administration of trabectedin and tend to regress spontaneously in about 15 days. If levels have not returned to normal after 21 days, a reduction or delay in the treatment is mandatory. Intravenous premedication with a corticosteroid such as dexamethasone is strongly recommended as an antiemetic and as a prophylaxis against hepatic toxicity. ${ }^{65}$ Some clinical studies have shown that co-treatment with steroids induces hepatic cytochrome P450 3A4 variant activity, reducing hepatic exposure to trabectedin and, consequently, the correlated hepatotoxicity. ${ }^{66,67}$

Rhabdomyolysis represents a potentially fatal adverse event related to the use of trabectedin. It occurs in $0.7 \%$ of cases, usually during the first 2 cycles of treatment, and has a $0.3 \%$ rate of fatal outcome. The event consists of skeletal muscle damage that releases muscle cell components into the bloodstream causing an increase in serum creatinine and creatine phosphokinase levels. ${ }^{68}$

Maintenance treatment with trabectedin has also been evaluated. A randomized French trial assessed the role of trabectedin maintenance therapy until disease progression in
885 patients with metastatic STS. Continuation of the drug was associated with longer PFS than early discontinuation of treatment, and showed an acceptable toxicity profile. ${ }^{69}$ On the basis of these results, Le Cesne et al carried out a prospective, randomized, phase II trial (T-DIS trial), confirming the improvement in PFS and OS in the continuation arm and the absence of cumulative toxicity. However, quality-of-life evaluations were not included in the trial. ${ }^{70}$ Overall, trabectedin is a drug with a favorable toxicity profile for the longterm treatment of STS, even in heavily pretreated patients.

\section{Trabectedin in current treatment guidelines}

Although the number of systemic therapies available for the treatment of STS has increased over the last decade, prognosis in the metastatic setting remains poor due to the limited efficacy of treatment options. The current gold standard firstline chemotherapy for advanced STS is doxorubicin and/or ifosfamide, but patients who progress during treatment have limited further options and a poor prognosis. ${ }^{5,8-11,30}$

Recently, a new drug, olaratumab, a platelet-derived growth factor receptor- $\alpha$-blocking antibody, was approved for use in association with doxorubicin as first-line therapy for patients with inoperable STS following evidence of improved survival with the combination compared to doxorubicin alone. ${ }^{71}$

The various clinical behaviors, the considerable molecular heterogeneity, and the different sensitivity to antitumor agents of STS are among the most important determinants of the lack of effective therapeutic approaches in these tumors. A better understanding of the underlying biology of the STS is undoubtedly needed.

Several phase II and III studies have reported an important activity of trabectedin for the treatment of patients with pretreated STS, leading to the approval of the drug in several countries. ESMO guidelines acknowledge trabectedin as one of the best options for second- or more-line therapy in

Table 2 Main prospective trials of trabectedin in STS patients

\begin{tabular}{|c|c|c|c|c|c|c|c|}
\hline Author & Phase & No of patients & Dose schedule & $\begin{array}{l}\text { Response } \\
\text { rate (\%) }\end{array}$ & $\begin{array}{l}\text { Median TTP } \\
\text { (months) }\end{array}$ & $\begin{array}{l}\text { 6-Month } \\
\text { PFS (\%) }\end{array}$ & $\begin{array}{l}\text { Median OS } \\
\text { (months) }\end{array}$ \\
\hline Yovine et $\mathrm{al}^{43}$ & II & 54 & $1.5 \mathrm{mg} / \mathrm{m}^{2} 24$-hour infusion $\mathrm{q} 3 \mathrm{w}$ & 4 & 1.9 & 24 & 12.8 \\
\hline Garcia-Carbonero et $\mathrm{al}^{44}$ & II & 36 & $1.5 \mathrm{mg} / \mathrm{m}^{2} 24$-hour infusion q3w & 8 & 1.7 & 23 & $\begin{array}{l}\text { I2.I (1-year } \\
\text { OS 53\%) }\end{array}$ \\
\hline Le Cesne et $\mathrm{al}^{45}$ & II & 104 & $1.5 \mathrm{mg} / \mathrm{m}^{2} 24$-hour infusion q3w & 8 & 3.4 & 29 & 9.2 \\
\hline Demetri et $\mathrm{al}^{13}$ & II & 270 & $\begin{array}{l}2 \text { arms: } \\
1.5 \mathrm{mg} / \mathrm{m}^{2} 24 \text {-hour infusion } \mathrm{q} 3 \mathrm{w} \\
0.58 \mathrm{mg} / \mathrm{m}^{2} 3 \text {-hour infusion } \\
\text { each week for } 3 \text { out of } 4 \text { weeks }\end{array}$ & $\begin{array}{l}5.6 \\
1.6\end{array}$ & $\begin{array}{l}3.7 \\
2.3\end{array}$ & $\begin{array}{l}35.5 \\
27.5\end{array}$ & $\begin{array}{l}13.9 \\
11.8\end{array}$ \\
\hline Demetri et $a^{13}$ & III & 518 & $1.5 \mathrm{mg} / \mathrm{m}^{2} 24$-hour infusion $\mathrm{q} 3 \mathrm{w}$ & 9.9 & I & 37 & 12.4 \\
\hline
\end{tabular}

Abbreviations: STS, soft tissue sarcoma; TTP, time to progression; PFS, progression-free survival; OS, overall survival; q3w, every 3 weeks. 
patients with metastatic LMS or LPS. However, the drug has also proven to be active in non-L-sarcoma histotypes, including TRS.

It has been seen that active second-line drugs are correlated with a 12 -week PFS in at least $40 \%$ of STS patients. ${ }^{72}$ Randomized and non-randomized trials have shown that trabectedin is associated with low response rates but prolonged tumor control, indicating its potential usefulness in second- and further-line treatment.

\section{Future perspectives}

A number of clinical trials are ongoing to explore the combination of chemotherapy or new molecules with trabectedin. Olaparib, an inhibitor of the enzyme poly(ADP-)ribose polymerase (PARP inhibitor), is currently being evaluated with trabectedin in a multicenter, open-label, non-randomized phase Ib study on metastatic sarcoma after failure of standard chemotherapy (NCT02398058). Another ongoing multicenter phase I trial is studying the efficacy and safety of trabectedin and metronomic cyclophosphamide in patients with advanced pretreated STS (NCT02805725).

\section{Conclusion}

The management of STS is a dynamic and complex process. The introduction of new molecules with innovative multiple mechanisms of action, such as trabectedin, into the therapeutic armamentarium of STS underlines the complexity and potential importance of immunogenicity and microenvironment interactions in the pathogenesis of STS. The unique antitumor activity of trabectedin consists not only in its cytotoxic activity, but also in its ability to modulate the tumor microenvironment. In several clinical trials, the use of trabectedin in L-sarcomas has led to an improvement in patient outcome. However, the drug has also proven feasible in a variety of nonL-sarcoma histotypes, including chromosomal translocated sarcomas such as synovial sarcoma and SFT. It is hoped that the use of biomarkers in the future will guide the treatment of STS, optimizing the use of available antitumor agents in an appropriate sequential or a combination strategy.

\section{Acknowledgment}

The authors thank Chiara Spadazzi for her help with the literature search and Cristiano Verna for editorial assistance.

\section{Author contributions}

All authors contributed toward data analysis, drafting and revising the paper and agree to be accountable for all aspects of the work.

\section{Disclosure}

The authors report no conflicts of interest in this work.

\section{References}

1. Siegel RL, Miller KD, Jemal A. Cancer statistics, 2015. CA Cancer J Clin. 2015;66(1):5-29.

2. Fletcher $\mathrm{CD}$. The evolving classification of soft tissue tumours - an update based on the new 2013 WHO classification. Histopathology. 2014;64(1):2-11.

3. Schwartz GK. Trabectedin and the L-Sarcomas: a decade-long odyssey. J Clin Oncol. 2016;34(8):769-771.

4. Ducimetière F, Lurkin A, Ranchère-Vince D, et al. Incidence of sarcoma histotypes and molecular subtypes in a prospective epidemiological study with central pathology review and molecular testing. PLoS One. 2011;6(8):e20294.

5. The ESMO/European Sarcoma Network Working Group. Soft tissue and visceral sarcomas: ESMO clinical practice guidelines for diagnosis, treatment and follow-up. Ann Oncol. 2014;25(Suppl 3):iii102-iii112.

6. Nussbaum DP, Rushing CN, Lane WO, et al. Preoperative or postoperative radiotherapy versus surgery alone for retroperitoneal sarcoma: a case-control, propensity score-matched analysis of a nationwide clinical oncology database. Lancet Oncol. 2016;17(7):966-975.

7. Mastrangelo G, Coindre JM, Ducimetière F, et al. Incidence of soft tissue sarcoma and beyond: a population-based prospective study in 3 European regions. Cancer. 2012;118(6):5339-5348.

8. Judson I, Verweij J, Gelderblom H, et al. Doxorubicin alone versus intensified doxorubicin plus ifosfamide for first-line treatment of advanced or metastatic soft-tissue sarcoma: a randomised controlled phase 3 trial. Lancet Oncol. 2014;15:415-423.

9. García-Del-Muro X, López-Pousa A, Maurel J, et al; Spanish Group for Research on Sarcomas. Randomized phase II study comparing gemcitabine plus dacarbazine versus dacarbazine alone in patients with previously treated soft tissue sarcoma: a Spanish group for research on sarcomas study. J Clin Oncol. 2011;29:2528-2533.

10. Le Cesne A, Antoine E, Spielmann M, et al. High-dose ifosfamide: circumvention of resistance to standard-dose ifosfamide in advanced soft tissue sarcomas. J Clin Oncol. 1995;13(7):1600-1608.

11. Van der Graaf WT, Blay JY, Chawla SP, et al. Pazopanib for metastatic soft-tissue sarcoma (PALETTE): a randomised, double-blind, placebocontrolled phase 3 trial. Lancet. 2012;379(9829):1879-1886.

12. Schöffski P, Maki R, Italiano A, et al. Randomized, open-label, multicenter, phase III study of eribulin versus dacarbazine in patients (pts) with leiomyosarcoma (LMS) and adipocytic sarcoma (ADI). J Clin Oncol. 2015;33.

13. Demetri GD, von Mehren M, Jones RL, et al. Efficacy and safety of trabectedin or dacarbazine for metastatic liposarcoma or leiomyosarcoma after failure of conventional chemotherapy: results of a phase III randomized multicenter clinical trial. J Clin Oncol. 2016;34(8): 786-793.

14. Demetri GD, Chawla SP, von Mehren M, et al. Efficacy and safety of trabectedin in patients with advanced or metastatic liposarcoma or leiomyosarcoma after failure of prior anthracyclines and ifosfamide: results of a randomized phase II study of two different schedules. J Clin Oncol. 2009;27(25):4188-4196.

15. Khalifa J, Ouali M, Chaltiel L, et al. Efficacy of trabectedin in malignant solitary fibrous tumors: a retrospective analysis from the French Sarcoma Group. BMC Cancer. 2015;15:700.

16. Le Cesne A, Cresta S, Maki RG, et al. A retrospective analysis of antitumour activity with trabectedin in translocation-related sarcomas. Eur J Cancer. 2012;48(16):3036-3044.

17. Sanfilippo R, Dileo P, Blay JY, et al. Trabectedin in advanced synovial sarcomas: a multicenter retrospective study from four European institutions and the Italian Rare Cancer Network. Anticancer Drugs. 2015; 26(6):678-681. 
18. De Sanctis R, Marrari A, Marchetti S, et al. Efficacy of trabectedin in advanced soft tissue sarcoma: beyond lipo- and leiomyosarcoma. Drug Des Devel Ther. 2015;27(9):5785-5791.

19. Singer S, Antonescu CR, Riedel E, Brennan MF. Histologic subtype and margin of resection predict pattern of recurrence and survival for retroperitoneal liposarcoma. Ann Surg. 2003;238(3):358-370.

20. Crago AM, Singer S. Clinical and molecular approaches to well differentiated and dedifferentiated liposarcoma. Curr Opin Oncol. 2011; 23(4):373-378.

21. Dei Tos AP, Doglioni C, Piccinin S, et al. Coordinated expression and amplification of the MDM2, CDK4 and HMGI-C genes in atypical lipomatous tumours. J Pathol. 2000;190(5):531-536.

22. Meis-Kindblom JM, Sjögren H, Kindblom LG, et al. Cytogenetic and molecular genetic analyses of liposarcoma and its soft tissue simulators: recognition of new variants and differential diagnosis. Virchows Arch. 2001;439(2):141-151.

23. Oliner JD, Pietenpol JA, Thiagalingam S, Gyuris J, Kinzler KW, Vogelstein B. Oncoprotein MDM2 conceals the activation domain of tumour suppressor p53. Nature. 1993;362(2):857-860.

24. Patel SR, Burgess MA, Plager C, Papadpoulos NE, Linke KA, Benjamin RS. Myxoid liposarcoma. Experience with chemotherapy. Cancer. 1994;74(4):1265-1269.

25. Crozat A, Aman P, Mandahl N, Ron D. Fusion of CHOP to a novel RNA-binding protein in human myxoid liposarcoma. Nature. 1993; 363(6430):640-644.

26. Dal Cin P, Sciot R, Panagopoulos I, et al. Additional evidence of a variant translocation $\mathrm{t}(12 ; 22)$ with EWS/CHOP fusion in myxoid liposarcoma: clinicopathological features. J Pathol. 1997;182(4):437-441.

27. Gebhard S, Coindre JM, Michels JJ, et al. Pleomorphic liposarcoma: clinicopathologic, immunohistochemical, and follow-up analysis of 63 cases: a study from the French Federation of Cancer Centers Sarcoma Group. Am J Surg Pathol. 2002;26(2):601-616.

28. Bathan AJ, Constantinidou A, Pollack SM, Jones RL. Diagnosis, prognosis, and management of leiomyosarcoma: recognition of anatomic variants. Curr Opin Oncol. 2013;25(4):384-389.

29. Farid M, Ong WS, Tan MH, et al. The influence of primary site on outcomes in leiomyosarcoma: a review of clinicopathologic differences between uterine and extrauterine disease. Am J Clin Oncol. 2013 36(4):368-374.

30. De Vita A, Mercatali L, Recine F, et al. Current classification, treatment options, and new perspectives in the management of adipocytic sarcomas. Onco Targets Ther. 2016;9(12):6233-6246.

31. Maki RG, Wathen JK, Patel SR, et al. Randomized phase II study of gemcitabine and docetaxel compared with gemcitabine alone in patients with metastatic soft tissue sarcomas: results of sarcoma alliance for research through collaboration study 002. J Clin Oncol. 2007; 25(4):2755-2763.

32. Zewail-Foote M, Hurley LH. Ecteinascidin 743: a minor groove alkylator that bends DNA toward the major groove. JMed Chem. 1999;42(8) 1493-1497.

33. Pommier Y, Kohlhagen G, Bailly C, Waring M, Mazumder A, Kohn KW. DNA sequence- and structure-selective alkylation of guanine N2 in the DNA minor groove by ecteinascidin 743, a potent antitumor compound from the Caribbean tunicate Ecteinascidia turbinata Biochemistry. 1996;35(41):13303-13309.

34. D'Incalci M, Galmarini CM. A review of trabectedin (ET-743): a unique mechanism of action. Mol Cancer Ther. 2010;9(8):2157-2163.

35. Friedman D, Hu Z, Kolb EA, Gorfajn B, Scotto KW. Ecteinascidin743 inhibits activated but not constitutive transcription. Cancer Res. 2002;62(12):3377-3381.

36. Forni C, Minuzzo M, Virdis E, et al. Trabectedin (ET-243) promotes differentiation in myxoid liposarcoma tumors. Mol Cancer Ther. 2009; 8(2):449-457.

37. Germano G, Frapolli R, Belgiovine C, et al. Role of macrophage targeting in the antitumor activity of trabectedin. Cancer Cell. 2013; 23(2):249-262.
38. Grosso F, Jones RL, Demetri GD, et al. Efficacy of trabectedin (ecteinascidin-743) in advanced pretreated myxoid liposarcomas: a retrospective study. Lancet Oncol. 2007;8(7):595-602.

39. Puchalski TA, Ryan DP, Garcia-Carbonero R, et al. Pharmacokinetics of ecteinascidin 743 administered as a $24-\mathrm{h}$ continuous intravenous infusion to adult patients with soft tissue sarcomas: associations with clinical characteristics, pathophysiological variables and toxicity. Cancer Chemother Pharmacol. 2002;50(4):309-319.

40. Ryan DP, Supko JG, Eder JP, et al. Phase I and pharmacokinetic study of ecteinascidin 743 administered as a 72-hour continuous intravenous infusion in patients with solid malignancies. Clin Cancer Res. 2001; $7(2): 231-242$.

41. Twelves C, Hoekman K, Bowman A, et al. Phase I and pharmacokinetic study of Yondelis (Ecteinascidin-743; ET-743) administered as an infusion over $1 \mathrm{~h}$ or $3 \mathrm{~h}$ every 21 days in patients with solid tumours. Eur J Cancer. 2003;39(13):1842-1851.

42. Taamma A, Misset JL, Riofrio M, et al. Phase I and pharmacokinetic study of ecteinascidin-743, a new marine compound, administered as a 24-hour continuous infusion in patients with solid tumors. J Clin Oncol. 2001;19(5):1256-1265.

43. Yovine A, Riofrio M, Blay JY, et al. Phase II study of ecteinascidin743 in advanced pretreated soft tissue sarcoma patients. J Clin Oncol. 2004;22(5):890-899.

44. Garcia-Carbonero R, Supko JG, Manola J, et al. Phase II and pharmacokinetic study of ecteinascidin 743 in patients with progressive sarcomas of soft tissues refractory to chemotherapy. J Clin Oncol. 2004; 22(8):1480-1490

45. Le Cesne A, Blay JY, Judson I, et al. Phase II study of ET-743 in advanced soft tissue sarcomas: a European Organisation for the Research and Treatment of Cancer (EORTC) soft tissue and bone sarcoma group trial. J Clin Oncol. 2005;23(3):576-584

46. Samuels BL, Chawla S, Patel S, et al. Clinical outcomes and safety with trabectedin therapy in patients with advanced soft tissue sarcomas following failure of prior chemotherapy: results of a worldwide expanded access program study. Ann Oncol. 2013;24(6):1703-1709.

47. Blay JY, Italiano A, Ray-Coquard I, et al. Long-term outcome and effect of maintenance therapy in patients with advanced sarcoma treated with trabectedin: an analysis of 181 patients of the French ATU compassionate use program. BMC Cancer. 2013;13:64.

48. Gronchi A, Bui BN, Bonvalot S, et al. Phase II clinical trial of neoadjuvant trabectedin in patients with advanced localized myxoid liposarcoma. Ann Oncol. 2012;23(3):771-776.

49. Gronchi A, Ferrari S, Quagliuolo V, et al. Full-dose neoadjuvant anthracycline + ifosfamide chemotherapy is associated with a relapse free survival (RFS) and overall survival (OS) benefit in localized highrisk adult soft tissue sarcomas (STS) of the extremities and trunk wall: interim analysis of a prospective randomized trial. Paper presented at: European Society for Medical Oncology (ESMO) 2016 Congress; October 7-11; 2016; Copenhagen.

50. Letson GD, Muro-Cacho CA. Genetic and molecular abnormalities in tumors of the bone and soft tissues. Cancer Control. 2001;8(3):239-251.

51. Geurts van Kessel A, Simons A, Comtesse PP, et al. Ring chromosomes in a malignant mesenchymoma. Cancer Genet Cytogenet. 1999; 109(2):119-122.

52. Ida K, Kobayashi S, Taki T, et al. EWS-FLI-1 and EWS-ERG chimeric mRNAs in Ewing's sarcoma and primitive neuroectodermal tumor. Int J Cancer. 1995;63(4):500-504.

53. Thway K, Noujaim J, Jones RL, Fisher C. Dermatofibrosarcoma protuberans: pathology, genetics, and potential therapeutic strategies. Ann Diagn Pathol. 2016;25:64-71.

54. Larramendy ML, Mandahl N, Mertens F, et al. Clinical significance of genetic imbalances revealed by comparative genomic hybridization in chondrosarcomas. Hum Pathol. 1999;30(10):1247-1253.

55. Mavrogenis AF, Bianchi G, Stavropoulos NA, Papagelopoulos PJ, Ruggieri P. Clinicopathological features, diagnosis and treatment of clear cell sarcoma/melanoma of soft parts. Hippokratia. 2013;17(4):298-302. 
56. Gleason BC, Hornick JL. Inflammatory myofibroblastic tumours: where are we now? J Clin Pathol. 2008;61(4):428-437.

57. Donner LR, Silva MT, Dobin SM, et al. Solitary fibrous tumor of the pleura: a cytogenetic study. Cancer Genet Cytogenet. 1999;111(2): 169-171.

58. Fricke A, Ullrich PV, Cimniak AF, et al. Synovial sarcoma microvesicles harbor the SYT-SSX fusion gene transcript: comparison of different methods of detection and implications in biomarker research. Stem Cells Int. 2016;2016:6146047.

59. Blay JY, Leahy MG, Nguyen BB, et al. Randomised phase III trial of trabectedin versus doxorubicin-based chemotherapy as first-line therapy in translocation-related sarcomas. Eur J Cancer. 2014;50(6): 1137-1147.

60. Kawai A, Araki N, Sugiura H, et al. Trabectedin monotherapy after standard chemotherapy versus best supportive care in patients with advanced, translocation-related sarcoma: a randomized, open-label, phase 2 study. Lancet Oncol. 2015;16(4):406-416.

61. Hoiczyk M, Grabellus F, Podleska L, et al. Trabectedin in metastatic soft tissue sarcomas: role of pretreatment and age. Int J Oncol. 2013; 43(1):23-28.

62. Chaigneau L, Kalbacher E, Thiery-Vuillemin A, et al. Efficacy of trabectedin in metastatic solitary fibrous tumor. Rare Tumors. 2011; 3(3):e29.

63. Bui-Nguyen B, Butrynski JE, Penel N, et al; European Organisation for Research and Treatment of Cancer Soft Tissue and Bone Sarcoma Group (EORTC/STBSG) and the Sarcoma Alliance for Research through Collaboration (SARC). A phase IIb multicentre study comparing the efficacy of trabectedin to doxorubicin in patients with advanced or metastatic untreated soft tissue sarcoma: the TRUSTS trial. Eur $J$ Cancer. 2015;51(10):1312-1320.

64. Martin-Broto J, Pousa AL, de Las Peñas R, et al. Randomized Phase II study of trabectedin and doxorubicin compared with doxorubicin alone as first-line treatment in patients with advanced soft tissue sarcomas: a Spanish group for research on sarcoma study. J Clin Oncol. 2016; 34(19):2294-2302.
65. Yondelis ${ }^{\circledR}$ summary of product characteristics. Available from: www. ema.europa.eu/docs/en_GB. Accessed March 3, 2016.

66. Grosso F, Dileo P, Sanfilippo R, et al. Steroid premedication markedly reduces liver and bone marrow toxicity of trabectedin in advanced sarcoma. Eur J Cancer. 2006;42(10):1484-1490.

67. Paz-Ares L, López-Pousa A, Poveda A, et al. Trabectedin in pre-treated patients with advanced or metastatic soft tissue sarcoma: a phase II study evaluating co-treatment with dexamethasone. Invest New Drugs. 2012;30(2):729-740.

68. Grosso F, D'Incalci M, Cartoafa M, et al. A comprehensive safety analysis confirms rhabdomyolysis as an uncommon adverse reaction in patients treated with trabectedin. Cancer Chemother Pharmacol. 2012; 69(6): 1557-1565

69. Le Cesne A, Ray-Coquard I, Duffaud F, et al; French Sarcoma Group. French Sarcoma Group. Trabectedin in patients with advanced soft tissue sarcoma: a retrospective national analysis of the French Sarcoma Group. Eur J Cancer. 2015;51(6):742-750.

70. Le Cesne A, Blay JY, Domont J, et al. Interruption versus continuation of trabectedin in patients with soft-tissue sarcoma (T-DIS): a randomised phase 2 trial. Lancet Oncol. 2015;16(3):312-319.

71. Tap WD, Jones RL, Van Tine BA, et al. Olaratumab and doxorubicin versus doxorubicin alone for treatment of soft-tissue sarcoma: an openlabel phase $1 \mathrm{~b}$ and randomised phase 2 trial. Lancet. 2016;388(10043): 488-497.

72. Van Glabbeke M, van Oosterom AT, Oosterhuis JW, et al. Prognostic factors for the outcome of chemotherapy in advanced soft tissue sarcoma: an analysis of 2,185 patients treated with anthracyclinecontaining first-line regimens - a European Organization for Research and Treatment of Cancer Soft Tissue and Bone Sarcoma Group Study. J Clin Oncol. 1999;17(1):150-157.
OncoTargets and Therapy

\section{Publish your work in this journal}

OncoTargets and Therapy is an international, peer-reviewed, open access journal focusing on the pathological basis of all cancers, potential targets for therapy and treatment protocols employed to improve the management of cancer patients. The journal also focuses on the impact of management programs and new therapeutic agents and protocols on

\section{Dovepress}

patient perspectives such as quality of life, adherence and satisfaction The manuscript management system is completely online and includes a very quick and fair peer-review system, which is all easy to use. Visit http://www.dovepress.com/testimonials.php to read real quotes from published authors. 